\title{
Marginal states of the resistive tearing mode with flow in cylindrical geometry
}

\author{
A. D. WOOD ${ }^{1}$, E. O'RIORDAN ${ }^{1}$, N. SWEENEY ${ }^{1}$ and R. B. PARIS ${ }^{2}$ \\ ${ }^{1}$ School of Mathematical Sciences and NCPST, Dublin City University, Dublin, Ireland \\ ${ }^{2}$ Division of Mathematical Sciences, University of Abertay Dundee, Dundee DD1 1HG, UK \\ (r.paris@abertay.ac.uk)
}

(Received 24 January 2003 and accepted 3 June 2003)

\begin{abstract}
The linear stability of tearing modes in a cylindrical plasma subject to a sub-Alfvénic equilibrium shear flow along the equilibrium magnetic field is considered. The equations in the resistive boundary layer at the rational surface are solved numerically using a Fourier transform combined with a finite-element approach. The behaviour of the growth rate as a function of the flow and the various parameters (including a perpendicular fluid viscosity) is obtained. Marginal stability curves showing the dependence of the familiar matching parameter $\Delta^{\prime}$ with flow and shear are also given.
\end{abstract}

\section{Introduction}

The first systematic investigations of resistive instabilities in the high conductivity limit were carried out in slab (Furth et al. 1963) and cylindrical (Coppi et al. 1966) geometries for static equilibria. Many laboratory and astrophysical plasmas, however, are found to be associated with equilibrium flows which can have a significant effect on the dynamics of the resistive instabilities. Considerable effort in recent years has been devoted to understanding the interaction between different regimes of equilibrium flow and the resistive modes.

The presence of a sub-Alfvénic shear flow along the confining magnetic field on the tearing mode in slab geometry was shown by Paris and Sy (1983) to have a destabilizing effect. In addition, it was established that the well-known stability condition $\Delta^{\prime}>0$ (where $\Delta^{\prime}$ is the stability parameter measuring the free energy available to drive the mode) can be modified to $\Delta^{\prime}>\Delta_{c}$, where $\Delta_{c} \leqslant 0$. Bondeson and Persson (1986) subsequently considered the effects of fluid viscosity and shear flow in the sub-Alfvénic regime and obtained marginal stability states for the plane tearing mode. More recent calculations in slab geometry, both analytical and numerical (Chen and Morrison 1990; Einaudi and Rubini 1986), have considered flows along the magnetic field comparable with the Alfvén speed, where it was found that in the external, ideal region such equilibrium flows can dramatically modify the value of the external matching parameter $\Delta^{\prime}$ to produce a strong stabilization of the tearing mode. Similar calculations have also been carried out in cylindrical geometry, both with and without equilibrium flow (Rosenblum 1984; Wessen and Perrson 1991), which demonstrate that the value of $\Delta^{\prime}$ in the outer region depends sensitively on the equilibrium current profile. 
The marginal states of the tearing and resistive interchange modes in cylindrical geometry, made possible by perpendicular and parallel viscosity, were investigated for a static equilibrium by Dagazian and Paris (1986) as a prerequisite for the calculation of anomalous heat transport in weakly nonlinear regimes. Here we extend these considerations in cylindrical geometry to include the effects of an equilibrium shear flow on the tearing mode only, as this is the most important resistive mode in tokomaks. The flow will again be assumed to be in the subAlfvénic regime, so that its effects are comparable with the basic tearing mode driving mechanism and the pressure gradient term. Our principal results concern the dependence of the instability growth rate on the various equilibrium parameters and the existence and nature of marginal stability curves corresponding to $\Delta^{\prime}<0$. We only retain the perpendicular viscosity, although parallel viscosity is found to exert a destabilizing influence on the flowless tearing mode (Dagazian and Paris 1986), since its effect is to reduce the stabilizing compressional coupling of sound wave propagation along the magnetic field (Iacono et al. 1994).

\section{The model}

We consider the model employed by Coppi et al. (1966) for the diffuse linear pinch specified by the equilibrium magnetic field $\mathbf{B}_{0}=\left\{0, B_{\theta}(r), B_{z}(r)\right\}$ and the equilibrium pressure gradient $-p_{0}^{\prime}(r)=B_{\theta}\left(r B_{\theta}\right)^{\prime} / r+B_{z} B_{z}^{\prime}$ in cylindrical $r, \theta, z$ coordinates. In the high conductivity limit we neglect the effect of the slow resistive diffusion in the radial direction and assume the presence of an equilibrium subAlfvénic shear flow $\mathbf{V}_{0}=\left\{0, V_{\theta}(r), V_{z}(r)\right\}$ directed along $\mathbf{B}_{0}$. The plasma is described by the resistive magnetohydrodynamic (MHD) set of equations in the limit of small resistivity $\eta$ and perpendicular viscosity $\mu_{\perp}$ given by

$$
\begin{gathered}
\rho \frac{d \mathbf{v}}{d t}=-\nabla p+\mathbf{J} \times \mathbf{B}-\mu_{\perp}\left(\nabla \times \nabla \times \mathbf{v}-\frac{4}{3} \nabla \nabla \cdot \mathbf{v}\right), \\
\frac{\partial \mathbf{B}}{\partial t}=\nabla \times(\mathbf{v} \times \mathbf{B})+\eta \nabla^{2} \mathbf{B}, \\
\nabla \cdot \mathbf{B}=0, \quad \nabla \times \mathbf{B}=\mathbf{J}, \\
\frac{\partial \rho}{\partial t}+\nabla \cdot(\rho \mathbf{v})=0, \quad \frac{d}{d t}\left(p \rho^{-\gamma}\right)=0 .
\end{gathered}
$$

We project all vectors in the three orthogonal directions $\mathbf{e}_{\mathrm{r}}, \mathbf{b}$ and $\mathbf{e}_{\mathrm{r}} \times \mathbf{b}$, where $\mathbf{b}=\mathbf{B}_{\mathbf{0}} / B, B=\left|\mathbf{B}_{\mathbf{0}}\right|$, and write linearized quantities such as $\tilde{\mathbf{B}}$ in the form $\tilde{\mathbf{B}}=\mathbf{e}_{\mathrm{r}} \tilde{B}_{\mathrm{r}}+$ $\mathbf{b} \tilde{B}_{\|}+\mathbf{e}_{\mathrm{r}} \times \mathbf{b} \tilde{B}_{\perp}$ and similarly for $\tilde{\mathbf{v}}$. Each component is assumed to vary like the single Fourier harmonic $\exp \{i(m \theta+k z)+\omega t\}$, with $\mathbf{k}=\{0, m / r, k\}$ and $\omega$ the growth rate. To simulate a large aspect ratio torus of major radius $\mathscr{R}_{0}$, we take $k=-n / \mathscr{R}_{0}$, where $m, n$ are integers. The rational surface $r=a$ is determined by $q(a)=m / n$, where $q(r)=r B_{z}(r) / \mathscr{R}_{0} B_{\theta}(r)$. The quantity $F=\mathbf{k} \cdot \mathbf{B}_{0}$ near the rational surface then takes the form $F \simeq F^{\prime}(a)(r-a)$ with $F^{\prime}(a)=-n B_{\theta}(a) q^{\prime}(a) / a$.

In terms of the resistive layer thickness $\delta_{\mathrm{R}}$, we introduce the variables $X=(r-a) /$ $\delta_{\mathrm{R}}, \omega_{\mathrm{R}}=\eta /\left(a \delta_{\mathrm{R}}\right)$ and $Q=\omega / \omega_{\mathrm{R}}$. The introduction of a perpendicular viscosity gives rise to two possible orderings: the first is the 'weak' viscous ordering associated with the scalings $\eta \sim \epsilon^{5}, \mu_{\perp} \sim \epsilon^{7}, V_{0} \sim \epsilon$ (where $\epsilon$ is a small dummy parameter) and

$$
\delta_{\mathrm{R}}=\left(\rho \eta^{2} / a F^{\prime 2}\right)^{1 / 5}, \quad \Gamma=\mu_{\perp} a /\left(\rho \eta \delta_{\mathrm{R}}\right),
$$


where $\rho \equiv \rho(a)$ and $F^{\prime} \equiv F^{\prime}(a)$. The radial component of the linearized magnetic field then has the scaling $\tilde{B}_{\mathrm{r}}=\epsilon^{4} \tilde{B}_{\mathrm{r}}^{(0)}+\epsilon^{6} \tilde{B}_{\mathrm{r}}^{(1)}+\ldots$, where $\tilde{B}_{\mathrm{r}}^{(0)}$ is constant across the resistive layer. We employ the standard scaled linearized variables

$$
\begin{aligned}
& \Xi \equiv \frac{\tilde{v}_{\mathrm{r}}}{\omega_{\mathrm{R}}}, \quad \Psi_{0} \equiv \frac{i \tilde{B}_{\mathrm{r}}^{(0)}}{\delta_{\mathrm{R}} F^{\prime}}, \quad \Psi_{1} \equiv \frac{i a \tilde{B}_{\mathrm{r}}^{(1)}}{\delta_{\mathrm{R}}^{2} F^{\prime}}, \\
& \Upsilon \equiv-\frac{2 k^{2} B}{\delta_{\mathrm{R}}\left(F^{\prime}\right)^{2}} \tilde{B}_{\|}, \quad W \equiv-\frac{2 i k^{2} B \delta_{\mathrm{R}}^{2}}{\eta F^{\prime}} \tilde{v}_{\|}
\end{aligned}
$$

and the parameters

$$
\begin{gathered}
S \equiv \frac{4}{\mathscr{R}_{0}^{2} q^{\prime 2}}, \quad D \equiv-\frac{2 q^{2} p_{0}^{\prime}(a)}{a B_{z}^{2} q^{\prime 2}}, \quad \hat{D} \equiv D \frac{a}{\delta_{\mathrm{R}}} \\
R \equiv\left(\mathbf{k} \cdot \mathbf{V}_{0}\right)^{\prime} \frac{\delta_{\mathrm{R}}}{\omega_{\mathrm{R}}}, \quad J \equiv-\frac{2 \sigma}{a \mathscr{R}_{0} q^{\prime}}, \quad J_{p} \equiv-\frac{a^{2}}{\mathscr{R}_{0} B_{\theta}^{2} q^{\prime}}\left(\mathbf{J}_{0} \cdot \mathbf{B}_{0}\right)^{\prime}
\end{gathered}
$$

where $\beta \equiv 2 p(a) / B^{2}, \sigma \equiv \mathbf{J}_{0} \cdot \mathbf{B}_{0} / B^{2}, B \equiv\left|\mathbf{B}_{0}(a)\right|, q \equiv q(a), q^{\prime} \equiv q^{\prime}(a)$, the equilibrium current terms, $J$ and $J_{p}$, are evaluated at $r=a$ and primes denote derivatives with respect to $r$.

The effect of the equilibrium shear flow in the resistive layer is to produce a Doppler shift on linearized quantities represented by

$$
\frac{\partial}{\partial t}+\mathbf{V}_{0} \cdot \nabla \equiv \omega+i \mathbf{k} \cdot \mathbf{V}_{0} \simeq \omega_{\mathrm{R}} Q_{*}, \quad Q_{*}=Q+i R X
$$

The resistive-layer equations about $r=a$ in the 'weak' viscous ordering then take the form (cf. Coppi et al. (1966, Appendix F)):

$$
\begin{gathered}
\Psi_{0}=\text { constant, } \quad \Psi_{1}^{\prime \prime}=X \Xi+Q_{*} \Psi_{0}, \\
\Gamma \Xi^{i v}=Q_{*} \Xi^{\prime \prime}-X^{2} \Xi+\Upsilon-Q_{*} X \Psi_{0}+J_{p} \Psi_{0}, \\
\Upsilon^{\prime \prime}=(S-2 \hat{D} / \gamma \beta) \Xi-X W, \\
\Gamma W^{\prime \prime}=Q_{*} W+X \Upsilon+(\hat{D}-J X) \Psi_{0},
\end{gathered}
$$

where the primes now denote differentiation with respect to $X$. The 'strong' viscous ordering is associated with the scalings $\eta \sim \mu_{\perp} \sim \epsilon^{3}, V_{0} \sim \epsilon$ and

$$
\delta_{\mathrm{R}}=\left(\rho \eta^{2} / F^{\prime 2}\right)^{1 / 6}, \quad \Gamma=\mu_{\perp} / \rho \eta
$$

together with the radial magnetic field given by $\tilde{B}_{\mathrm{r}}=\epsilon^{2} \tilde{B}_{\mathrm{r}}^{(0)}+\epsilon^{3} \tilde{B}_{\mathrm{r}}^{(1)}+\cdots$. In this case, the resulting set of equations in the resistive layer is described by (1)-(4) with the omission of the inertial terms $Q_{*} \Xi^{\prime \prime}$ and $Q_{*} W$ on the right-hand sides of (2) and (4), respectively.

We note that when $R=0$, the parameters $J$ and $J_{p}$ can be set to zero as these terms only affect the shape of the eigenfunctions and not the instability growth rate. When $R \neq 0$, however, these terms play a significant role in the calculation of the growth rate $Q$. In the following we assume that the pressure gradient term $D \sim \epsilon^{2}$ with $\beta$ kept finite, so that the term involving $\hat{D} / \gamma \beta$ in (3) may be neglected. 


\section{Numerical solution}

To solve the system (1)-(4) we introduce the Fourier transforms

$$
\begin{gathered}
\xi(k)=\int_{-\infty}^{\infty} \Xi(X) e^{-i k X} d X, \quad \hat{y}(k)=\int_{-\infty}^{\infty} \Upsilon(X) e^{-i k X} d X, \\
w(k)=-i \int_{-\infty}^{\infty} W(X) e^{-i k X} d X
\end{gathered}
$$

together with the new variables

$$
h(k)=\xi(k)+2 \pi i R \delta(k), \quad y(k)=\hat{y}(k)-2 \pi J \delta(k),
$$

where $\delta(k)$ denotes the delta function. We then obtain the system

$$
\begin{gathered}
L[h]+y=2 \pi \hat{J} \delta(k)+2 \pi i Q \delta^{\prime}(k), \\
w^{\prime}+k^{2} y+S h=2 \pi i R S \delta(k), \\
y^{\prime}+R k^{2} y+\left(Q+\Gamma k^{2}\right) w+R S h=2 \pi i D_{\mathrm{R}} \delta(k),
\end{gathered}
$$

where

$$
D_{\mathrm{R}} \equiv \hat{D}+S R^{2}, \quad \hat{J} \equiv-\left(J+J_{p}\right) \simeq \frac{a B^{2} q}{B_{\theta} B_{z} q^{\prime}} \sigma^{\prime}
$$

and $L$ denotes the viscous-tearing operator (Bondeson and Persson 1986; Hou et al. 1996):

$$
L[h] \equiv \frac{d^{2} h}{d k^{2}}+R \frac{d}{d k}\left(k^{2} h\right)-\left(Q k^{2}+\Gamma k^{4}\right) h .
$$

The calculation of the logarithmic jump $\Delta(Q)$ in the derivative of $\Psi$ across the resistive layer is obtained from (1) through use of the familiar constant- $\Psi$ approximation in the form

$$
\Delta(Q)=\int_{-\infty}^{\infty}\left(X \Xi+Q_{*} \Psi_{0}\right) d X,
$$

where without loss of generality we set $\Psi_{0}=1$. The transformed version of this matching condition then becomes

$$
\Delta(Q)=\frac{1}{2} i\left\{h^{\prime}(0-)+h^{\prime}(0+)\right\},
$$

where use has been made of the fact that the jump in $h(k)$ across $k=0$ is $2 \pi i Q$. The requirement $\Delta(Q)=\Delta^{\prime}$, where $\Delta^{\prime}$ (which is regarded as a parameter here) is the logarithmic jump in the derivative of $\Psi$ across $r=a$ from the outer solution, then determines the growth rate $Q$.

The eigenvalue $Q \equiv Q(R, \hat{J})$ satisfies the symmetry relations $Q(-R, \hat{J})=$ $Q(R,-\hat{J})=\bar{Q}(R, \hat{J})$, where $\bar{Q}$ denotes the complex conjugate, so that it is sufficient in the numerical calculations to consider $R \geqslant 0$ and $\hat{J} \geqslant 0$. The combination of (6) and (7) enables us to eliminate $w$ and to write the system (5)-(7) as the two coupled second-order differential equations

$$
\begin{gathered}
L[h]+y=2 \pi \hat{J} \delta(k)+2 \pi i Q \delta^{\prime}(k), \\
M[y]+S N[h]=-2 \pi i Q^{2} R S \delta(k)+2 \pi i Q D_{\mathrm{R}} \delta^{\prime}(k),
\end{gathered}
$$




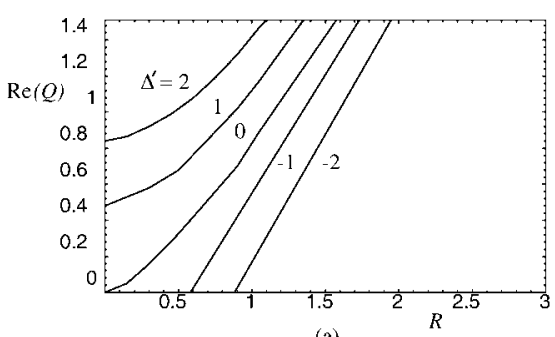

(a)

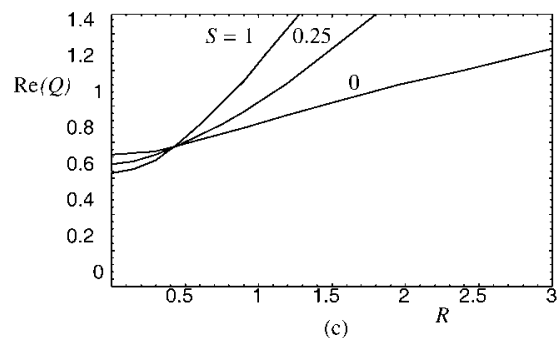

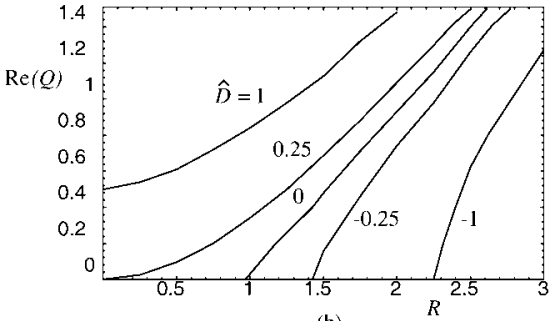

(b)

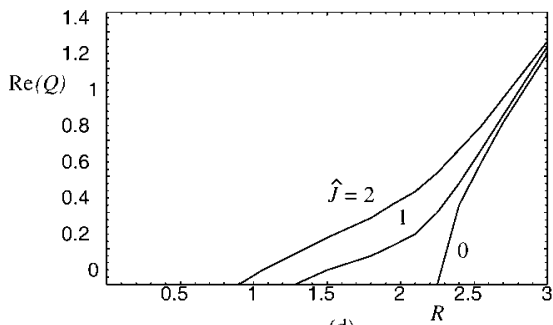

(d)

Figure 1. The behaviour of $\operatorname{Re}(Q)$ as a function of $R$ for $\Gamma=0.05$ and: (a) $S=1, \hat{D}=0, \hat{J}=0$ with varying $\Delta^{\prime}$; (b) $S=0.25, \hat{J}=0, \Delta^{\prime}=-1$ with varying $\hat{D}$; (c) $\hat{D}=0.25, \hat{J}=1, \Delta^{\prime}=1$ with varying $S$; and (d) $S=0.25, \hat{D}=-1, \Delta^{\prime}=-1$ with varying $\hat{J}$.

where $M$ and $N$ denote the operators

$$
\begin{gathered}
M[y] \equiv A \frac{d^{2} y}{d k^{2}}+\left(k^{2} A R-2 \Gamma k\right) \frac{d y}{d k}+\left(2 k R Q-k^{2} A^{2}\right) y, \\
N[h] \equiv R A \frac{d h}{d k}-\left(A^{2}+2 k \Gamma R\right) h, \quad A \equiv Q+\Gamma k^{2} .
\end{gathered}
$$

Dominant balance arguments applied to this coupled system show that $h(k)$ and $y(k)$ possess the leading behaviour $\exp \left[b_{ \pm} k^{3} / 3+c k\right]$ as $k \rightarrow \pm \infty$, where $b_{ \pm}=(-R \mp$ $\left.\left(R^{2}+4 \Gamma\right)^{1 / 2}\right) / 2$ and $c=Q /\left(R^{2}+4 \Gamma\right)^{1 / 2}$. The numerical solution of (9) and (10) is achieved by a standard finite-element method subject to the condition that $h(k)$ and $y(k)$ have finite norm. The infinite interval is replaced by the finite interval $[-L, L]$, for suitably chosen $L$, and the boundary conditions which are applied are

$$
\begin{gathered}
h^{\prime}( \pm L) / h( \pm L)=y^{\prime}( \pm L) / y( \pm L)=\alpha_{ \pm}, \\
\alpha_{ \pm}=\frac{1}{2}\left(-R \mp\left(R^{2}+4 \Gamma\right)^{1 / 2}\right) L^{2}+Q /\left(R^{2}+4 \Gamma\right)^{1 / 2},
\end{gathered}
$$

respectively, thus enabling the finite-element approximations to match onto the correct asymptotic behaviour of the solutions at the endpoints.

\section{Results and discussion}

We are primarily interested in situations with $\hat{D} \leqslant 0$ for which the interchange mode is suppressed. This can result either from peaked equilibrium current profiles or by the replacement of $\hat{D}>0$ in $(7)$ by $\hat{D}\left(1-q^{2}\right)$ (in a standard tokomak expansion) which is usually negative. We note that a key effect of the flow is to replace the pressure gradient term $\hat{D}$ by $D_{\mathrm{R}}=\hat{D}+S R^{2}$; a similar flow-induced modification to the interchange driving term has been found in the ideal MHD stability of cylindrical equilibria with mass flows (Bondeson et al. 1987). 


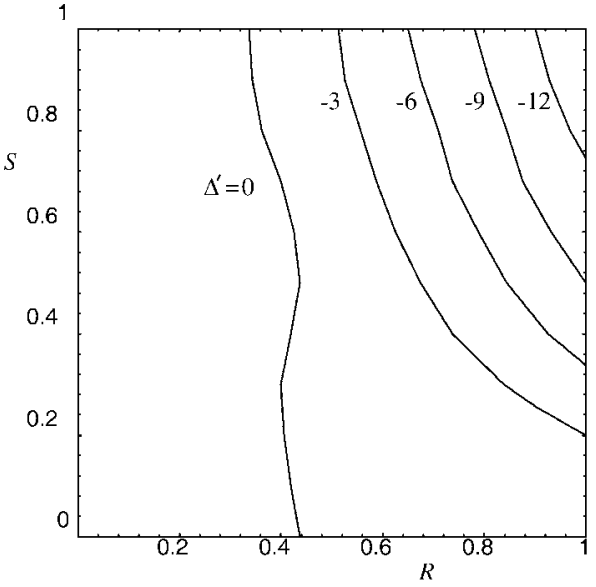

(a)

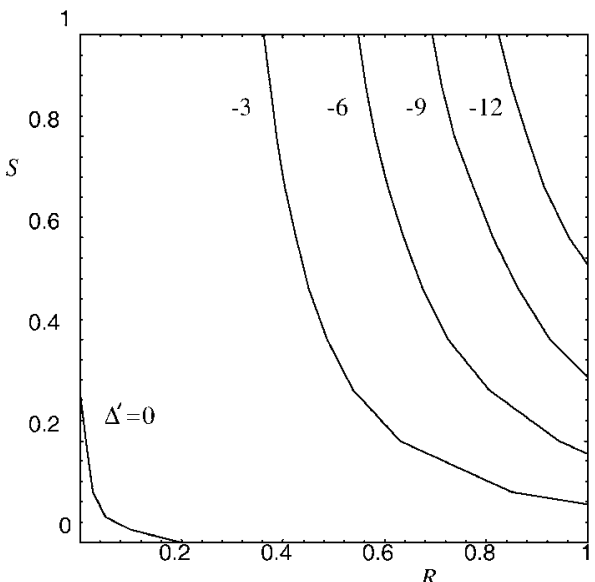

(c)

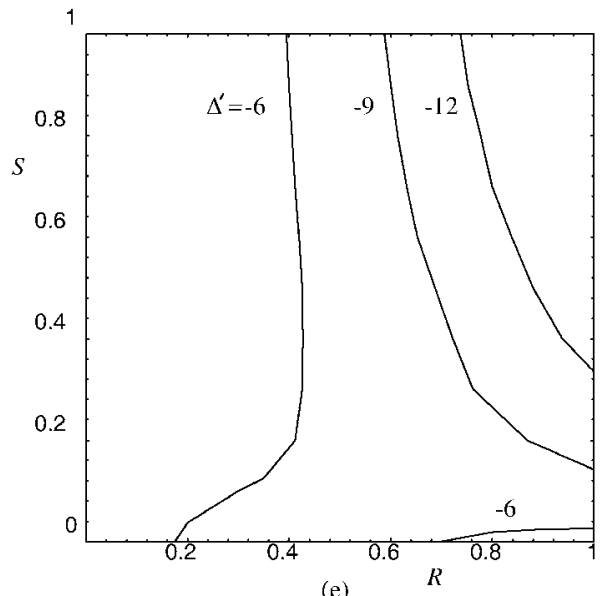

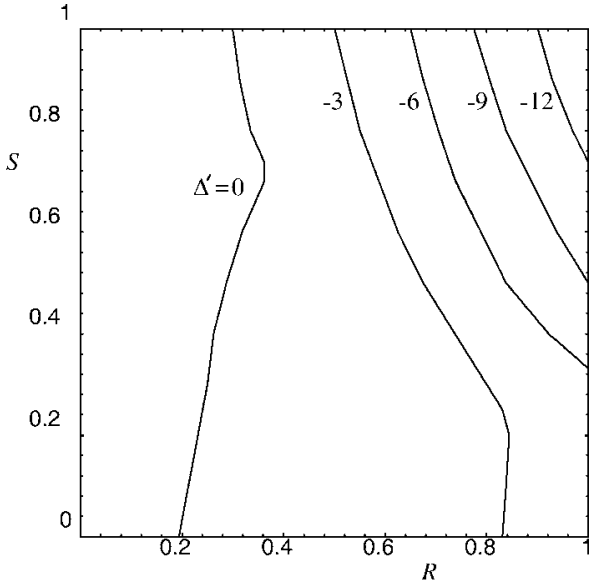

(b)

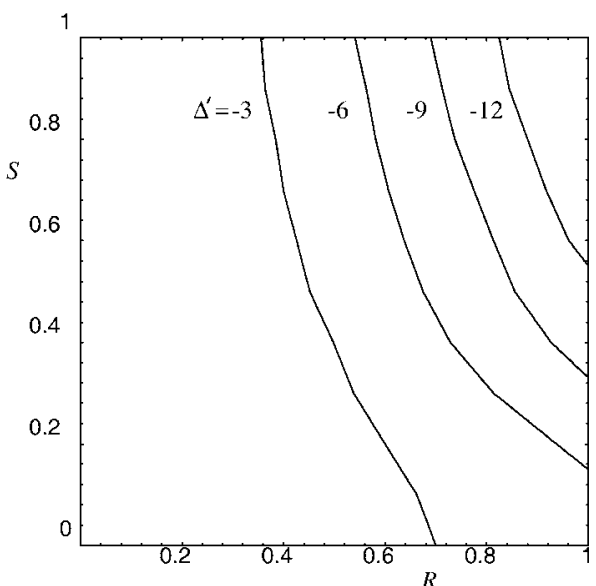

(d)

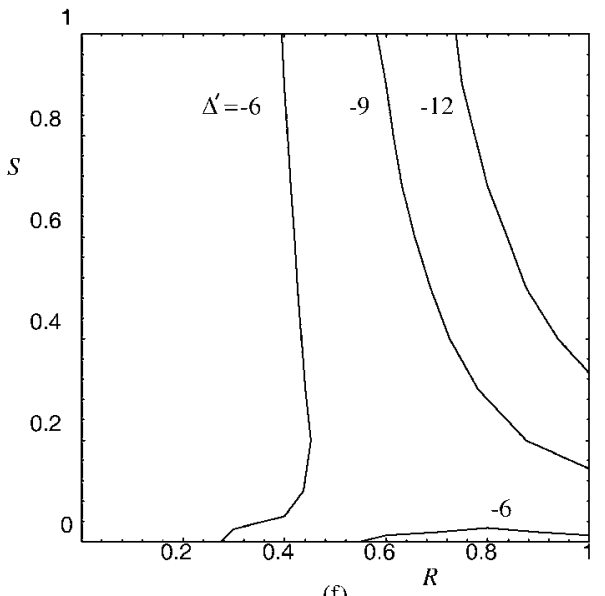

Figure 2. The marginal curves showing the behaviour of $\Delta^{\prime}$ when $\operatorname{Re}(Q)=0$ as a function of $R$ and $S$ for different values of the parameters $\hat{D}$ and $\hat{J}$ when $\Gamma=0.05$ : (a) $\hat{D}=-1$, $\hat{J}=0$; (b) $\hat{D}=-1, \hat{J}=1$; (c) $\hat{D}=0, \hat{J}=0$; (d) $\hat{D}=0, \hat{J}=1$; (e) $\hat{D}=1, \hat{J}=0$; and (f) $\hat{D}=1$, $\hat{J}=1$. 
We summarize the numerical results on the behaviour of $\operatorname{Re}(Q)$ as a function of the flow $R$ in Fig. 1, where the typical dependence on the parameters $\Delta^{\prime}, \hat{D}, S$ and $\hat{J}$ is displayed. In all cases it can be seen that the presence of the flow is destabilizing. In Fig. 1(a) and (b) we show the variation in $\operatorname{Re}(Q)$ for a range of values of $\Delta^{\prime}$ that corresponds to the tearing mode in the absence of flow being unstable $\left(\Delta^{\prime}>0\right)$ or stable $\left(\Delta^{\prime}<0\right)$, and in situations in which the pressure gradient term $\hat{D}$ is either stabilizing or destabilizing. In Fig. 1(c) it can be seen that the growth rate sensitively depends on the shear parameter $S$ (due to the dependence of $D_{\mathrm{R}}$ ), while in Fig. $1(\mathrm{~d})$ once $R \gtrsim 2$, where the effective pressure gradient term $D_{\mathrm{R}}$ changes sign, $\operatorname{Re}(Q)$ is relatively insensitive to the current parameter $\hat{J}$. Although not displayed, the variation of the growth rate curves on the viscosity parameter $\Gamma$ was found to be small.

In Fig. 2 we present a set of marginal curves in the $R-S$ plane showing the critical value of $\Delta^{\prime}$ for onset of instability $\operatorname{Re}(Q)=0$ when the viscosity parameter $\Gamma=0.05$. For the pure tearing mode $(\hat{D}=0)$, the marginal condition when $R=0$ in the inviscid limit is the well-known value $\Delta^{\prime}=0$, independent of $S$, which corresponds to the $S$-axis; the modification of the curve $\Delta^{\prime}=0$ in Fig. $2(\mathrm{c})$ and $(\mathrm{d})$ is due to $\Gamma \neq 0$. For $R>0$, we require $\Delta^{\prime}<0$ to yield a marginal state reflecting the destabilizing nature of the flow. In situations with $\hat{D}<0$ in Fig. 2(a) and (b), the curves of constant $\Delta^{\prime}$ are displaced to the right where, for small values of $R$, marginal states can exist for $\Delta^{\prime}>0$. When $\hat{D}>0$ the reverse situation is the case, see Fig. $2(\mathrm{e})$ and (f). For larger values of $R$ and $S \simeq 1$, the marginal curves are seen to possess a similar structure on account of the dominance of the term $S R^{2}$ in the flow-induced pressure gradient term $D_{\mathrm{R}}$.

\section{References}

Bondeson, A., Iacono, R. and Bhattacharjee, A. 1987 Phys. Fluids 30, 2167.

Bondeson, A. and Persson, M. 1986 Phys. Fluids 29, 2997.

Chen, X. L. and Morrison, P. J. 1990 Phys. Fluids B2, 495.

Coppi, B., Greene, J. M. and Johnson, J. L. 1966 Nucl. Fusion 6, 101.

Dagazian, R. Y. and Paris, R. B. 1986 Phys. Fluids 29, 762.

Einaudi, G. and Rubini, F. 1986 Phys. Fluids 29, 2563.

Furth, H. P., Killeen, J. and Rosenbluth, M. N. 1963 Phys. Fluids 6, 459.

Hou, L., Paris, R. B. and Wood, A. D. 1996 Phys. Plasmas 3, 473.

Iacono, R., Bhattacharjee, A., Ronchi, C., Greene, J. M. and Hughes, M. H. 1994 Phys. Plasmas 1, 2645.

Paris, R. B. and Sy, W. N.-C. 1983 Phys. Fluids 26, 2966.

Rosenblum, M. 1984 J. Plasma Phys. 31, 447.

Wessen, K. P. and Persson, M. 1991 J. Plasma Phys. 45, 267. 